\title{
Évaluer plutôt que noter
}

Jacques Weiss

\section{(2)enEdition}

Journals

Édition électronique

URL : http://journals.openedition.org/ries/3264

DOI : 10.4000/ries.3264

ISSN : 2261-4265

\section{Éditeur}

Centre international d'études pédagogiques

\section{Édition imprimée}

Date de publication : 1 septembre 1996

Pagination : 22-34

ISSN : 1254-4590

\section{Référence électronique}

Jacques Weiss, "Évaluer plutôt que noter », Revue internationale d'éducation de Sèvres [En ligne],

11 | 1996, mis en ligne le 29 juillet 2013, consulté le 30 avril 2019. URL : http://

journals.openedition.org/ries/3264 ; DOI : 10.4000/ries.3264

Ce document a été généré automatiquement le 30 avril 2019

(c) Tous droits réservés 


\title{
Évaluer plutôt que noter
}

\author{
Jacques Weiss
}

\section{NOTE DE L'AUTEUR}

L'auteur de cet article remercie les membres du Groupe romand et tessinois d'évaluation pour leurs réflexions et leurs apports

1 Mettre des notes, ne pas en mettre ? Telle est la question! Mais est-ce bien là une bonne question? Et pourquoi se poser cette question? Noter finalement, est-ce évaluer ou seulement une façon d'exprimer les résultats de l'évaluation?

2 Nous montrerons que la notation chiffrée n'est pas l'évaluation, à peine l'une de ses manifestations, la plus visible peut-être, mais pas la plus opportune. L'évaluation ne peut se réduire à la mise de notes, elle est davantage un comportement inhérent à l'apprentissage, nécessaire à l'enseignement et à la gestion d'un système de formation.

\section{La note, forme visible de l'évaluation et miroir aux alouettes}

3 La notation chiffrée est commode, apparemment facile à lire et à comprendre. Chiffrée, jusqu'aux décimales quelquefois, elle est considérée généralement comme précise, juste, indiscutable. C'est ce qui fait sa force, sa résistance au temps. Elle rassure en effet parents, élèves, jusqu'aux enseignants eux-mêmes qui voient en elle une précision mathématique bienvenue dans une école tellement conflictuelle, tellement complexe. Ces notes sont toutefois bien commodes pour prendre des décisions administratives, pour stimuler le travail des élèves ou pour maitriser la gestion de la classe.

4 L'analyse la plus superficielle montre pourtant combien elle est inadéquate et trompeuse. L'évidence est telle qu'en faire la démonstration serait faire injure au lecteur. Chacun sait combien l'usage de chiffres est abusif et combien la précision des évaluations est illusoire. 
Depuis de nombreuses années, les insuffisances de la notation chiffrée sont dénoncées et critiquées ${ }^{1}$.

Des propositions ont été faites pour la mise en œuvre d'autres formes d'évaluation, et des expériences ont été lancées. Il n'en demeure par moins que les innovations sont encore timides, et les changements, modestes. Les orientations sont toutefois données. Nous allons en exposer les principes et les illustrer par plusieurs innovations réalisées à différents niveaux de formation.

\section{Une évaluation pour qui, pour quoi, comment ?}

6 Au-delà de la question de la notation, il importe de s'interroger en priorité sur le sens de l'évaluation en milieu scolaire et de nous demander pour qui évaluer, pour quoi et comment le faire. L'évaluation remplit en effet plusieurs fonctions et se réalise sous des formes différentes selon le destinataire ou l'usager :

- elle est formative quand elle tend à favoriser les apprentissages des élèves; elle est informative (ou prévisionnelle) quand elle permet aux familles de prendre les décisions les plus judicieuses ;

- elle est indicative quand elle met à la disposition des enseignants des repères nationaux ou régionaux;

- elle est externe quand elle donne aux responsables administratifs des indicateurs susceptibles de permettre le pilotage du système de formation ;

- elle est certificative enfin quand elle atteste aux yeux de la société les compétences, les savoirs et les savoir-faire du diplômé au terme de sa formation.

- Une seule procédure d'évaluation ne peut remplir toutes ces fonctions. Les procédures doivent au contraire être différentes et conçues spécifiquement. Nous allons le montrer de manière différenciée selon les bénéficiaires ou les usagers de l'évaluation.

\section{Une évaluation formative pour les élèves}

7 La fonction première du système de formation est de favoriser les apprentissages des élèves. L'évaluation formative est également soumise à cet impératif.

8 L'évaluation formative est une pratique pédagogique constitutive de l'enseignement luimême.

9 Elle « fait, avant tout, partie intégrante du processus éducatif normal, les "erreurs" étant à considérer comme des moments dans la résolution d'un problème (plus généralement comme des moments dans l'apprentissage)... $»^{2}$.

10 "L'évaluation formative sert à l'amélioration, à l'orientation et au contrôle du processus d'apprentissage $»^{3}$.

11 Elle favorise la régulation des apprentissages et la différenciation des enseignements en fonction des rythmes d'acquisition des élèves. Dans le cas de l'évaluation formative, l'élève est amené à repérer et à comprendre ses erreurs pour ne plus les commettre à l'avenir et pour réorienter ses apprentissages. Les didacticiens du français et des mathématiques ont particulièrement développé cette conception de l'évaluation formative .

12 L'évaluation formative est interactive. Elle est centrée sur l'apprenant, mais elle doit être aussi soutenue et animée par l'enseignant selon une pratique appelée « auto-évaluation 
guidée ». Elle favorise la prise de conscience par l'élève de ses progrès ou de ses compétences par l'analyse et l'appréciation de la qualité de ses travaux scolaires effectifs en fonction des objectifs du programme ainsi que des critères de "suffisance» ou en fonction des termes de son "contrat didactique »; le rôle de l'enseignant est en effet décisif dans le guidage des autoévaluations; il suggère les apprentissages complémentaires à effectuer; il modère les surestimations; il réajuste les sousestimations. C'est donc bien là une évaluation et un enseignement interactifs, faits de réajustements successifs et de confrontations aux objectifs pédagogiques et à leurs exigences.

13 Pour illustrer ce que peut être une pratique de l'évaluation formative en classe, nous présenterons d'une part celle qui a cours dans des séquences didactiques de français, d'autre part celle qui prévaut dans la constitution d'un portfolio et dans l'appréciation des documents qu'il contient.

\section{L'évaluation dans les séquences didactiques de français}

Les séquences didactiques de français ont pour but d'aider les élèves à apprendre à s'exprimer en recourant à divers genres d'écrits dans des situations de communication qui font sens. Ces séquences comprennent trois phases :

- la rédaction d'un premier texte qui permet la manifestation des compétences des élèves avant l'apprentissage et l'identification par l'enseignant des problèmes d'écriture de ses élèves dans cette situation ;

- la réalisation de diverses activités d'écriture liées au genre de texte étudié (texte informatif, argumentatif, narratif) et aux problèmes identifiés lors du prétexte ;

- la réécriture du premier texte en fonction des apprentissages effectués lors de la deuxième étape.

15 Ce travail de production-apprentissage-réécriture illustre la démarche d'une évaluation formative intégrée à l'apprentissage. Le prétexte permet d'identifier les problèmes et de fixer les objectifs de l'apprentissage. Ces premiers textes peuvent faire l'objet d'évaluation de groupe, d'appréciations réciproques. Ce travail prépare les activités par modules qui caractérisent la deuxième étape. Au cours de cette phase, les élèves sont amenés à étudier et à analyser des textes ou discours authentiques propres au genre. Ils en dégagent ensemble, avec l'enseignant, les caractéristiques propres afin de pouvoir les mettre en œuvre à leur tour au moment de la réécriture de leur texte. Au cours de cette phase d'apprentissage, les élèves élaborent également en commun des grilles de contrôle ou des aide-mémoire, sorte de documents de synthèse des différentes observations réalisées au cours de la deuxième étape. L'aide-mémoire prend le plus souvent la forme d'une liste d'objectifs à atteindre, de constats, ou de règles à observer au moment de la production.

16 «La grille de contrôle - ou l'aide-mémoire - a pour fonction importante de susciter l'autorégulation chez les élèves; en effet, tout au long de la séquence, ces derniers savent quels sont les acquis les plus déterminants qu'ils doivent maitriser; de plus, au moment de la production finale, ils se réfèrent à la grille de contrôle qui les aide ainsi à améliorer leur performance et à prendre conscience des progrès accomplis. De son côté, l'enseignant, grâce à cette grille, est en mesure d'évaluer les apprentissages réalisés. Le fait de la construire avec ses élèves exige de lui qu'il adopte un langage partagé par la classe; en outre, cela lui permet de se concentrer sur les objectifs les plus importants 
qu'il désire atteindre avec ses élèves. Cet instrument est donc doublement autorégulateur : pour les élèves et pour l'enseignant ${ }^{5}$ ».

\section{L'évaluation par le portfolio}

17 Comme alternative à l'évaluation chiffrée, le portfolio ou dossier d'évaluation est expérimenté ou appliqué dans de nombreuses écoles aux États- Unis, particulièrement dans le Vermont, au Canada, en Australie, aux Pays-Bas, en Angleterre. Il est censé favoriser la pratique de l'auto-évaluation et de la méta-cognition ${ }^{6}$ et permettre le développement de processus d'anticipation, de contrôle, d'ajustement qui mènent à l'autorégulation ${ }^{7}$ Pour Arter et Spandel $^{8}$, le portfolio est

un ensemble de travaux significatifs de l'élève qui donne un aperçu de ses efforts, de ses progrès ou de sa performance dans une ou plusieurs matières. Le choix des éléments constitutifs doit faire appel à la participation de l'élève et inclure les critères de sélection, les directives qui permettent de juger de la valeur des réalisations et des exemples d'autorégulation de l'élève9

Pour Simon \& Forgette-Giroux ${ }^{10}$, le portfolio est

un recueil cumulatif et continu d'indicateurs du cheminement de l'élève dans ses apprentissages, sélectionnés et commentés par l'élève et l'enseignant à des fins d'évaluation.

19 Une expérience d'évaluation formative au moyen $d u$ portfolio a été réalisée à Genève dans une classe multi-âges de vingt-quatre élèves (10-12 ans) ${ }^{11}$. Les auteurs de cette recherche constatent que les élèves ont appris à trier parmi le matériel utilisé, à choisir les productions représentatives de leurs forces et faiblesses, à argumenter et à assumer leurs choix, à entrer dans une analyse de leurs activités scolaires, à confronter leurs avis à ceux de l'enseignant, etc. Cette étude a montré également que l'élaboration d'un portfolio favorise l'autonomie. Par la pratique du contrat, les élèves ont été amenés à assumer leurs choix, gérer leur temps, à travailler selon leurs possibilités. La pratique du portfolio, organisatrice et régulatrice des apprentissages de l'élève, relève bien de l'évaluation formative.

\section{Une évaluation informative (prévisionnelle) pour l'enfant et ses parents}

20 L'évaluation pour la famille est informative. Elle fournit à l'élève et à ses parents les informations nécessaires aux prises de décisions éducatives qui leur reviennent : méthode personnelle de travail, choix d'éventuels appuis offerts par l'école ou hors d'elle, choix des cours ou filières de formation les mieux adaptés aux compétences ou aspirations de l'enfant.

21 Cette évaluation se construit autour de l'échange entre, d'un côté, un ou des enseignants, de l'autre, l'enfant et ses parents ${ }^{12}$. Les appréciations de l'enseignant, inscrites dans un bulletin scolaire, et les travaux de l'élève réunis dans un dossier d'évaluation, constituent la base de ces échanges qui ont lieu à l'occasion d'entretiens individuels. Nous présentons ci-dessous un projet de bulletin scolaire répondant à cette exigence de communication d'informations générales aux parents. Nous examinerons ensuite le rôle et le fonctionnement d'un entretien d'évaluation tel qu'il est expérimenté dans une région alémanique de la Suisse, et comment, à cette occasion, le portfolio peut soutenir cette évaluation collective. 


\section{Le bulletin d'information} les invite à l'échange en cas de difficultés majeures ou de décisions à prendre. Les expériences réalisées au Québec depuis plus de vingt ans dans ce domaine (Conseil supérieur de l'éducation, 1992) ont montré que les bulletins descriptifs (liste d'objectifs détaillés) ne répondaient pas aux attentes des parents qui souhaitaient des informations écrites plus générales et davantage d'échanges et de contacts directs. Un bulletin devrait être de lecture aisée, simple et de consultation rapide de manière à donner aux parents une première idée d'ensemble de la situation scolaire, mais suffisante pour les alerter en cas de problèmes scolaires majeurs de leur enfant. Caille ${ }^{13}$ a montré que les contacts spontanés des familles avec l'enseignant étaient en rapport avec leur perception de l'urgence.

Le service des didactiques de la partie francophone du canton de Fribourg a mené, durant cinq ans, une étude auprès des enseignants, des parents et des inspecteurs scolaires afin de concevoir un nouveau bulletin scolaire répondant mieux aux exigences des familles. Lors de la mise en circulation d'une version antérieure du livret scolaire, les auteurs avaient notamment constaté que l'attribution de notes, au premier semestre déjà, constituait une incohérence, puisqu'il était admis que les enfants disposaient de toute une année pour atteindre les objectifs définis par le programme. Le terme du semestre n'est en effet qu'une occasion de faire un bilan provisoire, un état de la situation des apprentissages et de l'attitude en classe, bilan assurément utile pour d'éventuels réajustements, mesures d'appui, ou entretiens avec l'enseignant.

Les résultats scolaires se réfèrent aux objectifs du plan d'études. La note est, dans ce cas, le résultat d'une évaluation globale basée sur des épreuves, des observations et une appréciation générale ${ }^{14}$.

Un espace est laissé à disposition de l'enseignant afin qu'il puisse nuancer et expliciter le jugement porté sur le travail, le comportement et l'attitude de l'élève. A la fin de l'année, les parents retirent la page du bulletin qui traite des comportements en classe afin d'éviter tout effet « d'étiquetage ».

L'évaluation exprimée dans le bulletin ne détermine nullement la réussite ou l'échec de l'année. Elle n'a pas de rôle sélectif. Il est admis, dans ce projet de réforme, que «l'enfant parcourt le cycle primaire en principe en six ans. Il est promu à la fin de l'année dans la classe supérieure. S'il est régulièrement en retard ou s'il est en avance par rapport au programme, il y a lieu de prendre des mesures d'aide pédagogique particulières ». Les auteurs du projet de réforme des procédures d'évaluation recommandent en outre que la décision d'une éventuelle répétition de l'année soit prise en commun entre les parents et l'enseignant et qu'en cas de désaccord, l'enseignant tranche en prenant en considération l'intérêt de l'enfant.

\section{L'entretien d'évaluation}

27 L'entretien d'évaluation représente une autre forme de communication de l'information scolaire à la famille. Cette pratique est courante dans certains pays. Au Danemark, l'information est communiquée aux parents exclusivement sous forme verbale jusqu'au niveau 8. A partir de ce niveau, elle l'est par oral et par écrit. La Norvège n'accorde que peu d'importance à l'évaluation formelle avant le niveau 7 ; l'accent est mis sur l'auto-

Revue internationale d'éducation de Sèvres, 11 | 1996 
évaluation des élèves ainsi que sur l'entretien avec les familles. Parmi les formes de communication des résultats des évaluations aux familles, plusieurs innovations ont été en effet entreprises dans différents pays, et notamment dans plusieurs cantons de la Suisse alémanique. Il s'agit du remplacement de l'information écrite par un ou plusieurs entretiens individuels avec la famille et en présence de l'enfant.

28 À l'occasion d'entretien(s) individualisé(s), les enseignants (et quelquefois l'élève, comme au Québec) commentent et apprécient avec les familles les progrès accomplis et la qualité des travaux sélectionnés par l'élève, travaux constitutifs du dossier de travaux ou du portfolio et représentatifs des compétences du moment. Ces entretiens ont pour but d'aider les élèves à s'évaluer et de conseiller les familles dans le choix des mesures les plus favorables à prendre, pédagogiques par différenciation interne, ou structurelles par différenciation externe, pour construire le meilleur itinéraire scolaire de l'enfant.

Ces activités de conseil et de guidage sont de la responsabilité professionnelle de l'enseignant au même titre que l'enseignement, et comme l'une des tâches inscrites à son cahier de charge. Il est en effet de la responsabilité de l'enseignant de guider l'élève et ses parents, de les stimuler dans l'élaboration d'un projet de formation à la fois ambitieux et réaliste. Les enseignants revendiquent d'ailleurs ce statut au nom de la professionnalisation de leur activité. Leur tâche d'enseignement s'enrichit ainsi de celle de soutenir, par l'information et le conseil, les apprentissages de l'élève et les choix des itinéraires de formation. Les appréciations faites ainsi en commun par l'enseignant avec l'élève lui-même et avec ses parents permettent alors de mesurer les risques et de prendre les décisions donnant les meilleures chances à l'élève.

Cette tâche est effectivement importante pour encadrer les appréciations des familles, et tenter de réduire les inégalités sociales. Duru-Bellat et Mingat (1992) ont montré que les biais sociaux intervenaient fortement dès que la prise de décision de l'orientation était laissée aux familles, les milieux aisés prenant des risques que les familles modestes ne prenaient pas. Des entretiens réguliers et obligatoires sont donc à instaurer et à instituer, particulièrement avec les familles les moins informées des enjeux et des règles de fonctionnement du système de formation. L'entretien avec la famille peut alors être considéré comme une mesure visant à réduire les inégalités sociales. Une étude de Vallet \& Caille ${ }^{15}$ montrait en effet que les familles émigrées avaient des ambitions élevées de formation pour leurs enfants, et que ces ambitions pouvaient se voir réalisées grâce au dialogue institué avec les familles lors des procédures d'orientation. Quel que soit le niveau social des familles, ces chercheurs constatent en effet que les enfants de ces familles d'émigrés conseillés par les enseignants effectuent de meilleurs parcours scolaires que les enfants des familles françaises. Des chercheurs du cycle d'orientation de Genève, qui ont constaté une relativement faible sélection sociale au cours du cycle secondaire, pensent pouvoir attribuer également ce fait à l'importante structure d'aide et de conseils mise à la disposition des élèves ${ }^{16}$.

\section{Une évaluation indicative pour l'enseignant}

31 La mise en œuvre d'une évaluation formative et informative qui confère à l'enseignant un rôle majeur dans la régulation des apprentissages et des orientations des élèves peut comporter, en dépit des précautions prises, des risques de biais majeurs par l'absence de points de repère ou de critères externes de référence suffisamment précis (objectifs et seuils de suffisance). En l'absence de tels repères extérieurs, les enseignants adoptent 
ceux qu'ils ont à proximité : la moyenne de la classe considérée comme norme, ou les attentes des enseignants des degrés suivants. Il n'est plus nécessaire de rappeler les critiques faites à cette évaluation normative, selon laquelle la référence des évaluations individuelles est la "classe de l'année » ou le niveau d'un établissement, et non les objectifs du programme. Des recherches belges ${ }^{17}$ ont clairement mis en évidence certaines tendances des enseignants à l'hypernormativité par anticipation des objectifs ou exigences du degré suivant. Critiquer cette pratique ne suffit pas cependant, car les objectifs des plans d'études sont à l'évidence insuffisamment précis pour être véritablement des instruments de référence permettant un jugement équitable des niveaux atteints par les élèves. Il appartient donc à l'institution scolaire de fournir à l'enseignant des données de référence, des balises externes le mettant en mesure de situer le niveau de sa classe. Il lui revient également de le préparer à le faire. À l'occasion d'une évaluation nationale (belge) externe effectuée auprès de 50000 élèves de $5^{\mathrm{e}}$ rimaire ${ }^{18}$, si $61 \%$ des enseignants belges reconnaissent comme important de pouvoir situer les résultats de leur classe par rapport à la moyenne des classes, ils ne sont en effet que $51 \%$ à l'avoir fait.

32 La direction de l'Évaluation et de la Prospective (DEP), en France, fournit de telles informations depuis 1989. Elle met à la disposition des enseignants des classes de CE2, de $6^{e}$ et de seconde, au début de chaque année, des protocoles d'évaluation constitués d'un référentiel (tableau des capacités-compétences-objectifs) et d'un ensemble d'items assortis aux rubriques du référentiel. Un logiciel leur permet de saisir et d'analyser les données. Il fournit également des conseils pour l'interprétation et l'exploitation des résultats. Des «banques d'items" peuvent en outre être consultées librement en cours d'année. Comme le fait remarquer Allai ${ }^{19}$,

(ces instruments) favorisent donc la mise en œuvre d'une évaluation formative tout en permettant un ajustement des objectifs et des seuils d'exigence de la classe à des objectifs et exigences externes, nationales ou régionales.

Ces repères externes constituent pour l'enseignant un moyen de contrôler sa propre marge d'erreur ${ }^{20}$ et permettent un ajustement des «représentations personnelles »du programme de chaque enseignant - c'est-à-dire les objectifs et les critères qu'il s'est fixés pour sa classe -, aux « représentations collectives » - c'est-à-dire les objectifs et critères définis par le groupe des auteurs des épreuves.

Ces épreuves établies au niveau d'un pays ou d'une région ne couvrent qu'une partie du programme. Compte tenu de ces limites, il importe donc de les compléter et de les renouveler périodiquement.

\section{Une évaluation externe pour le pilotage de l'institution}

35 Un système de formation doit pouvoir disposer de procédures de contrôle et de régulation - d'évaluation formative appliquée à l'organisation elle-même en quelque sorte. Il appartient en effet à ses acteurs de vérifier si les finalités arrêtées et les objectifs fixés sont effectivement tenus, si les moyens mis en œuvre et les structures répondent aux besoins de la formation, si les didactiques appliquées conviennent aux conceptions éducatives retenues.

Pour remplir ces missions, les institutions scolaires se dotent généralement de services d'évaluation ou de recherche, chargés de prélever les données nécessaires et d'élaborer des propositions d'ajustement ou des correctifs. Depuis 1987, l'Éducation nationale 
française a mis en place une direction de l'Évaluation et de la Prospective dont les missions sont «de connaître, d'évaluer et de prévoir ce qui se rapporte au système éducatif, à son fonctionnement et à ses résultats. » L'Espagne a créé en 1993 un Institut national pour la qualité et l'évaluation (INCE). Au cours de ces vingt-cinq dernières années, la Suisse romande et les cantons de la Suisse latine se sont dotés progressivement de tels dispositifs d'évaluation et de recherche (plus de soixante chercheurs répartis en une dizaine d'instituts décentralisés) chargés de remplir des missions analogues, enrichies de celles de proposer des orientations pédagogiques, des didactiques et des supports à l'enseignement et à l'apprentissage ${ }^{21}$.

37 Ces évaluations - qui peuvent prendre la forme de passation d'épreuves auprès des élèves - produisent des résultats utiles pour les responsables de la formation, les cadres de tout niveau et les enseignants. Ils ne portent souvent que sur quelques objectifs ciblés, choisis en fonction de priorité d'étude et ne proposent pas les mêmes items à tous les élèves ou à toutes les classes. Ils ne sont en effet pas conçus pour apprécier les compétences individuelles des élèves.

\section{Une évaluation certificative pour la société}

L'évaluation certificative remplit une fonction sociale particulière par l'attestation officielle des formations acquises par l'apprenant au terme d'une période de formation. Il s'agit de la reconnaissance de compétences globales correspondant à une unité ou crédit de formation dont l'articulation avec d'autres unités ou crédits peut constituer une compétence plus générale encore, professionnelle par exemple. Ces évaluations portent sur des réalisations mettant en œuvre des compétences complexes, intégratives, correspondant soit à des objectifs-noyaux, soit à des objectifs terminaux d'intégration, soit encore à des socles de compétences. Il peut s'agir de la rédaction d'un mémoire, d'une argumentation, de la résolution d'un problème en scolarisation générale, ou du traitement d'un cas ou encore de la production d'objets en scolarisation professionnelle. L'évaluation certificative peut prendre différentes formes selon qu'il s'agit d'attester une compétence générale (capacité d'expression, d'argumentation, de raisonnement) ou une compétence professionnelle (capacité d'enseigner, de soigner, de réparer, de construire).

Les « certifications sur travaux » sont fréquemment utilisées en formation d'adultes. Elles mettent en évidence un profil personnel de compétences avec ses points forts et ses faiblesses. L'université de Louvain-la-Neuve vient d'opter pour la suppression des examens universitaires de dernière année de médecine et propose "une évaluation globale au cours de laquelle, dans un premier temps, l'étudiant présentera l'articulation et l'évolution de son projet de formation, ainsi que ce qu'il a réalisé au cours de sa formation en médecine générale, et dans un second temps, il devra gérer un cas de médecine générale abordant ainsi les différents objectifs de la formation. La préparation à ce jury se fera tout au long de la formation, au cours de tutorat, sur base d'un "carnet de formation", reprenant les négociations concernant le projet de formation de l'étudiant ; plaçant ainsi l'évaluation formative comme un moteur de la formation ${ }^{22}$. Une conception similaire de l'évaluation est appliquée à l'École de soins infirmiers de la République et Canton du Jura ${ }^{23}$. Le dossier de formation y est défini comme « un recueil du parcours de l'élève. C'est lui qui le constitue, le conserve et en a l'entière responsabilité. Il y introduit à sa manière, avec ses moyens personnels, les moments-clefs 
de la formation, ses forces, ses faiblesses, les progrès établis, les moyens d'aide choisis... Le tuteur peut, à la demande de l'élève, y collaborer ».

On parlera plutôt d'évaluation sommative provisoire ou de bilan intermédiaire dans le cas des formations de type scolaire ne représentant qu'une étape vers une maîtrise finale plus lointaine.

\section{La révolution copernicienne de l'évaluation}

41 Le système complexe des notes et des moyennes trouve pour une large part sa raison d'être dans la nécessité face à laquelle se trouvent les responsables de l'institution scolaire et les enseignants de devoir justifier les décisions qu'ils sont amenés à prendre et qui déterminent réussite ou échec des élèves, choix de filières prometteuses ou plus limitées. La garantie d'égalité de traitement et d'équité des jugements doit dans ce cas pouvoir être démontrée en cas de contestation des familles. L'abstraction du chiffre, la précision des valeurs décimales est généralement considérée comme un rempart contre la critique et comme une preuve de la justesse de l'évaluation et de ses conséquences scolaires.

Cette notation, que l'on sait imparfaite et trompeuse, tente toutefois d'approcher la précision et l'objectivité par une « calculation » complexe et un lourd travail de la part de l'enseignant: élaboration de nombreuses "épreuves » ou "contrôles ", constitution de barêmes de cotation, codification, calcul de moyennes. Dans le secondaire, ces pratiques, cumulées pour chaque discipline, provoquent en outre un "roulement " incessant d'évaluations pour l'élève qui deviennent l'objectif premier de son travail scolaire (130 épreuves par élève et par an en moyenne au cycle d'orientation de Genève).

Pour échapper à cette mécanique dont on voit mal les finalités formatives, il convient de décharger fortement l'institution de formation de sa responsabilité concernant l'avenir scolaire de l'élève. Cette responsabilité doit revenir à l'enfant et à sa famille. Par une pratique régulière de la mise en perspective des compétences de l'élève, de ses projets et des offres de formation faites par l'institution, les familles devraient se trouver en mesure de prendre finalement elles-mêmes les décisions les plus adéquates, sur la base d'appréciations mûries et fondées. Dans cette perspective d'échanges et de responsabilité assumée, nous osons les trois propositions suivantes:

- tout d'abord un renversement des responsabilités des décisions où, sauf cas exceptionnels, les choix des itinéraires de formation seraient confiés aux familles auxquelles l'institution scolaire, par ses enseignants, aurait dispensé recommandations et conseils, éventuellement insistants ;

- ensuite l'attribution d'une valeur indicative et non décisive aux notes obtenues (si elles devaient être conservées) ; la décision d'un redoublement de classe par exemple serait alors prise après discussion et appréciation des chances et des risques par les familles avec l'enseignant ;

- enfin la réduction des occasions de choix majeurs et «de carrière " (redoublement, orientation en filières hiérarchisées) par l'introduction de cycles longs de formation, pouvant même s'étendre à l'ensemble de la scolarité au cours de laquelle l'évaluation pourrait prendre des formes plus qualitatives et formatives.

- Ces propositions mériteraient d'être examinées de près et vérifiées par l'observation d'écoles ou d'établissements qui expérimentent ou qui appliquent déjà quelques-unes de ces pratiques nouvelles d'évaluation. 


\section{NOTES}

1. Jean Cardinet, Où mènent les recherches sur la notation scolaire?, Mons, Université de MonsHainaut, Institut d'administration scolaire, 1995.

2. G. De Landsheere, Dictionnaire de l'évaluation et de la recherche en éducation, Paris, Presses universitaires de France (PUF), 1992.

3. SIPRI, «Appréciation du travail des élèves », in : J.-P. Salamin (éd.), École primaire suisse : une école pour apprendre: 22 thèses pour le développement de l'école primaire: projet SIPRI, Berne, Conférence suisse des directeurs cantonaux de l'Instruction publique (CDIP), 1986, p. 83-106.

4. D. Bain, «L'évaluation formative fait fausse route : de la nécessité de changer de cap », Mesure et évaluation en éducation, 1988, 10 (4), p. 23-32.

F. Jaquet (à paraître), "Situation-problème en mathématiques et évaluation formative ", in : J. Weiss (réd.), Vers une conception cohérente de l'évaluation pour la scolarité obligatoire en Suisse romande et au Tessin, Neuchâtel, Institut romand de recherches et de documentation pédagogiques (IRDP).

5. M. Wirthner (à paraître), «L'évaluation formative dans les séquences didactiques de français ", in : J. Weiss (réd.), op. cit.

6. B. Noël, La métacognition, Bruxelles, De Bceck-Wesmael, 1991.

J.-L. Wolfs, "Contribution à l'opérationnalisation du concept de métacognition », Recherche et éducation : théorie et pratique, 1992, 10 (3), p. 3-13.

B. Noël, M. Romainville \& J.-L. Wolfs, «La métacognition : facettes et pertinence du concept en éducation ", Revue française de pédagogie, 1995, 112, 47-56.

7. L. Allal « Régulations métacognitives : quelle place pour l'élève dans l'évaluation formative? ", in: L. Allai, D. Bain \& P. Perrenoud (éds), Évaluation formative et didactique du français, Neuchâtel, Delachaux \& Niestlé, 1993, p. 81-97.

8. J.-A. Arter \& V. Spandel, « Using portfolios of student work in instruction and assessment: an MCME instruction module ", Education measurement: issues and pratices, 1992, 11 (1), p. 36-44.

9. Traduction libre de M. Simon \& R. Forgette-Giroux, "Vers une utilisation rationnelle du dossier d'apprentissage ", Mesure et évaluation en éducation, 1994, 16 (3/4), p. 27-40.

10. Simon \& Forgette-Giroux, op. cit.

11. E. Wegmuller (à paraître), « Le portfolio : le dossier d'élève ", in : J. Weiss (réd.), op. cit.

12. J. Weiss (éd.), L.'évaluation: un problème de communication, Neuchâtel, Institut romand de recherches et de documentation pédagogiques (IRDP), Cousset, Delval, 1991.

13. J.-P. Caille, «Les parents d'élèves de collège et les études de leur enfant : attentes et degré », Éducation et formations, 1992, n 32, p. 15-23.

14. Les notes ont les significations suivantes : 6 = l'enfant maîtrise les objectifs du programme. Il est capable d'utiliser les acquisitions et habiletés exigées en diverses situations d'une manière autonome. 5 = l'enfant maîtrise les objectifs du programme. Il a de bonnes connaissances et des habiletés durables. $4=$ l'enfant ne satisfait qu'un minimum des objectifs du programme (fundamentum). Les connaissances et les habiletés sont considérées comme instables. 3 = l'enfant n'a pas atteint les objectifs du programme. Les connaissances et habiletés requises n'ont pas été intégrées. 2 et 1 ne sont pas utilisés dans le livret scolaire. Des mesures pédagogiques sont prises dans les cas problématiques.

15. L.-A. Vallet \& J.-P. Caille, « Les carrières scolaires au collège des élèves étrangers ou issus de l'immigration ", Éducation \& formations, 1995, n 40, p. 5-14. 
16. F. Rastoldo, «Modèle (s) actuel(s) d'orientation/sélection », in : F. Gabriel (éd.),... nous on s'en fout, on est en G..., (p. 53-71), Genève, Centre de recherches psychopédagogiques du cycle d'orientation (CRPP), 1995.

17. A. Grisay, "Que peut-on "prescrire" en matière d'évaluation-bilan? ", in : J.-M. de Ketele (éd.), L'évaluation : approche descriptive ou prescriptive?, Bruxelles, De Boeck-Wesmael (Pédagogies en développement. Problématiques et recherches), 1986, p. 161-178.

18. M. Herphelin, «Bilan de la première opération d'évaluation externe menée en Communauté française, cinquième année primaire, année scolaire 1994/1995: épreuve standardisée en mathématique et en français ", in : Pilotinfo, 1996, 12.

19. L. Allal (à paraître), «Une instance d'évaluation centralisée peut-elle servir à une prise de décision décentralisée ? », in : Actes des journées du PNR33 des 30 et 31 mars 1995.

20. J. Cardinet, Remettre le quantitatif à sa place en évaluation scolaire, Neuchâtel, Institut romand de recherches et de documentation pédagogiques (IRDP) (Recherches 90.101), 1990.

21. R. Hutin \& J. Weiss, Chercher ensemble : 20 ans de recherches coordonnées au service de l'école, Neuchâtel, Institut romand de recherches et de documentation pédagogiques (IRDP), Le Mont-sur-Lausanne, Loisirs et pédagogie (LEP), 1994.

22. Information de M.N. De Theux, coordinatrice pédagogique, du 14 juillet 1995.

23. P. Chételat, J. Gury \& J.-CI. Richoz, «Conception pédagogique de l'évaluation », in : L'arbre à projets : une expérience pédagogique dans l'enseignement des soins infirmiers, Delémont, École de soins infirmiers de la République et Canton du Jura, 1993, p. 165-183.

\section{RÉSUMÉS}

L'évaluation scolaire joue des rôles différents dans la société : formative pour l'élève, informative pour les parents, indicative pour l'enseignant. Elle ne peut donc pas se réduire à une note chiffrée et cette note n'en est pas le véhicule le plus pertinent. D'autres outils - portfolio, entretien - permettent de responsabiliser les différents partenaires de l'évaluation, selon le rôle que l'on attribue à celle-ci.

\section{INDEX}

Index géographique : France, Suisse

Mots-clés : évaluation, notation, évaluation de l'élève

\section{AUTEUR}

\section{JACQUES WEISS}

Chef du service de la recherche

Institut romand de recherches et de documentation pédagogiques Neuchâtel, Suisse 